\title{
Medial Palpebral Ligament
}

National Cancer Institute

\section{Source}

National Cancer Institute. Medial Palpebral Ligament. NCI Thesaurus. Code C52724.

A fibrous band located on the eyelid that connects the medial ends of the tarsal plates with the maxilla at the medial edge of the orbit. 